\title{
Diversidad taxonómica y funcional en la comunidad de peces de la pesca de arrastre de camarón en el norte del Golfo de California, México
}

\author{
Eloísa Herrera-Valdivia ${ }^{1,2}$, Juana López-Martínez ${ }^{1 *}$, Sergio Castillo Vargasmachuca ${ }^{2} \&$ Alma \\ Rosa García-Juárez ${ }^{3}$ \\ 1. Centro de Investigaciones Biológicas del Noroeste, S.C. Km 2.35 Carretera a Las Tinajas, S/N Colonia Tinajas. \\ Guaymas, Sonora, México. C. P. 85460; jlopez04@cibnor.mx, eloisa04@cibnor.mx \\ 2. Universidad Autónoma de Nayarit. Posgrado en Ciencias Biológico Agropecuarias, Carretera Tepic-Compostela Km. \\ 9, Xalisco Nayarit, C.P. 63780, México; sergio_machuca_@hotmail.com \\ 3. Centro Regional de Investigación Pesquera Ensenada. INAPESCA. Carretera Tijuana-Ensenada, Km. 97.5 El Sauzal \\ de Rodríguez, B.C., México; ayaxa@hotmail.com \\ * Correspondencia
}

Recibido 16-IX-2015. Corregido 15-XII-2015. Aceptado 29-I-2016.

\begin{abstract}
Taxonomic and functional diversity of the bycatch fishes community of trawl fishing from Northern Gulf of California, Mexico. The Northern Gulf of California (NGC) is a mega diverse area of high endemism with major economic interest because of the multi-specific fisheries developed, mainly shrimp. There is a lack of recent studies on bycatch fish assemblages, so during the fishing seasons from 2009-2010 and 2010-2011, on board 13 shrimp boats, 14 commercial fishing trips were performed from $5 \mathrm{~m}-90 \mathrm{~m}$ in depth with a total of 119 catches. The 119 catches were analyzed to assess fish community structure using taxonomic diversity indices to detect changes in the community following the taxonomic distinctness average $\Delta+$ and the diversity index $\Delta^{*}$ (TAXDEST of the PRIMER v6 program). To confirm the structure of functional groups, we considered similarities of ecologic and morphologic traits among species. The results showed that the indices $\Delta+$ and $\Delta^{*}$ were within the expected average and confidence intervals at $95 \%$, finding significant differences between the indices. The analyses showed a well-structured community because of the great variety of forms and functions of the species within the community. In the community of the functional groups, reproduction was the ecological attribute that contributed the most to their structure. The community structure was represented by intermediate trophic levels (3-3.9), preferably primary and secondary carnivores within the trophic categories of predators of benthic ichthyo-fauna that belong to demersal species of soft bottoms and mostly fusiform body. To conclude, the NGC showed high functional redundancy according to the estimated functional groups, thus the ecosystem was considered stable and with great diversity. This type of studies should be followed using fishing and environmental effort due to the great biological and ecologic importance in the area. Rev. Biol. Trop. 64 (2): 587-602. Epub 2016 June 01.
\end{abstract}

Key words: fish, Northern Gulf of California, functional diversity, taxonomic diversity.

Las investigaciones orientadas a la evaluación de los efectos de la pesca con enfoque ecosistémico son relativamente recientes e involucran la relación directa entre la biodiversidad y el funcionamiento de los ecosistemas (Fulton, Spith, \& Punt, 2005; Gristina, Bahri. Fiorentino, \& Garofalo, 2006). En este contexto, los estudios de diversidad funcional definida como el número de grupos funcionales formados por los rasgos morfológicos, fisiológicos o fenológico llamados caracteres funcionales, son relacionados con uno o más procesos ecológicos y a diferentes respuestas a factores ambientales (Rosenfeld, 2002). Son representados por las especies dentro de una comunidad natural; facilitando los análisis comparativos entre especies. Al encontrarse mayor número de grupos funcionales en la comunidad, la 
interacción entre grupos será mayor, lo que se traduce en una comunidad con capacidad para absorber alteraciones manteniendo su misma estructura y funcionamiento (Walker et al., 2004). Así mismo, la presencia de especies dominantes y subordinadas dentro de los grupos funcionales aumenta la redundancia, esto es, aquellas especies que realizan la misma función (Naeem, Lawler, Thompson, Lawton, \& Woodfin, 1994; Rosenfeld, 2002).

Por otro lado, Warwick y Clarke (1995, 1998) y Clarke y Warwick (1998, 1999, 2001), introdujeron los índices de diversidad taxonómica para detectar cambios taxonómicos en las comunidades de peces. Estos índices consideran el peso de las diferencias taxonómicas entre las especies: Así, el índice de distinción taxonómica promedio $\Delta+$ y el índice de variación taxonómica promedio $\Lambda+$ se basan en presencia/ausencia y son independientes del tamaño de la muestra y del esfuerzo del muestreo, relacionados con la diversidad funcional (Clarke \& Warwick, 1998; Clarke \& Warwick, 2001). Estos índices son importantes porque permiten comparar la distribución de especies a escala espacio-temporal (Nieto, 2010; Palacios, 2011; Escobar, 2012).

El Golfo de California (GC), considerado como uno de los ecosistemas marinos de mayor productividad en el mundo, posee gran diversidad de especies, relacionada directamente con la estabilidad y resiliencia del ecosistema (Palacios, 2011), con una alta variedad topográfica y batimétrica, que proporciona un mayor número de hábitats para peces y otros organismos marinos (López, Hernández, Herrera, Rábago, \& Morales, 2007). Hacia la parte norteña del GC, se encuentra el área de estudio, el Norte del Golfo de California (NGC). Este cuenta con una superficie estimada en 7200 $\mathrm{km}^{2}$ (Nelson et al., 1980) y una profundidad promedio de $200 \mathrm{~m}$. El área comprende desde el Delta del Río Colorado hacia la región de las grandes islas: Isla Tiburón y Ángel de la Guardia. Más al norte se encuentra la Reserva de la Biosfera Alto Golfo de California y Delta del Río Colorado RBAGCyDC, de la cual $82 \%$ lo constituye la zona de amortiguamiento
(DOF, 1993) lugar donde existe gran actividad pesquera multiespecífica, dentro de ellas la del camarón. Esta pesquería es fuente constante de controversia entre conservación y aprovechamiento, al ser fuertemente señalada como causa de disturbio a los fondos marinos por su alteración en los ecosistemas demersales. Existen escasas evaluaciones sobre las implicaciones ecológicas que tienen las actividades pesqueras sobre las comunidades explotadas, específicamente sobre las comunidades de peces que son afectadas por la pesca de arrastre. Es por ello crucial conocer y evaluar los grupos funcionales del componente íctico que forman parte de la fauna de acompañamiento del camarón FAC en el norte del Golfo de California.

\section{MATERIAL Y MÉTODOS}

La composición taxonómica de los peces del NGC se obtuvo de 119 arrastres con red de pesca comercial, realizados a profundidades entre 9-90 m (Fig. 1), en 14 viajes de pesca de camarón a bordo de 13 embarcaciones de la flota comercial durante las temporadas de pesca 2009-2010 y 2010-2011 (cada temporada estuvo compuesta de septiembre a marzo). Además, incluyó información obtenida de un crucero prospectivo llevado a cabo en un periodo de veda en agosto 2011, en la misma zona y con la misma arte de pesca que los arrastres comerciales. Para los análisis se obtuvo muestras al azar, de aproximadamente $20 \mathrm{~kg}$ de la FAC; el tamaño de muestra se determinó siguiendo los criterios estándar (Box, Stuart, \& Hunter, 2008).

De las muestras así recolectadas, se llevó a cabo la identificación de las especies de peces, para lo cual se utilizaron las claves de Jordan \& Evermann (1893-1900), Norman (1934), Curran (1942), Zahuranec (1967), Miller \& Lea (1972), Castro-Aguirre (1978), Compagno (1984), Fischer et al. (1995). La bibliografía especializada para algunos grupos incluyó: Castro-Aguirre \& Espinosa-Pérez (1996) en rayas; McPhail (1958) para la familia Sciaenidae, en Pleuronectiformes; Ginsburg (1958), Rosenblatt \& Johnson (1974) para Diplectrum, 


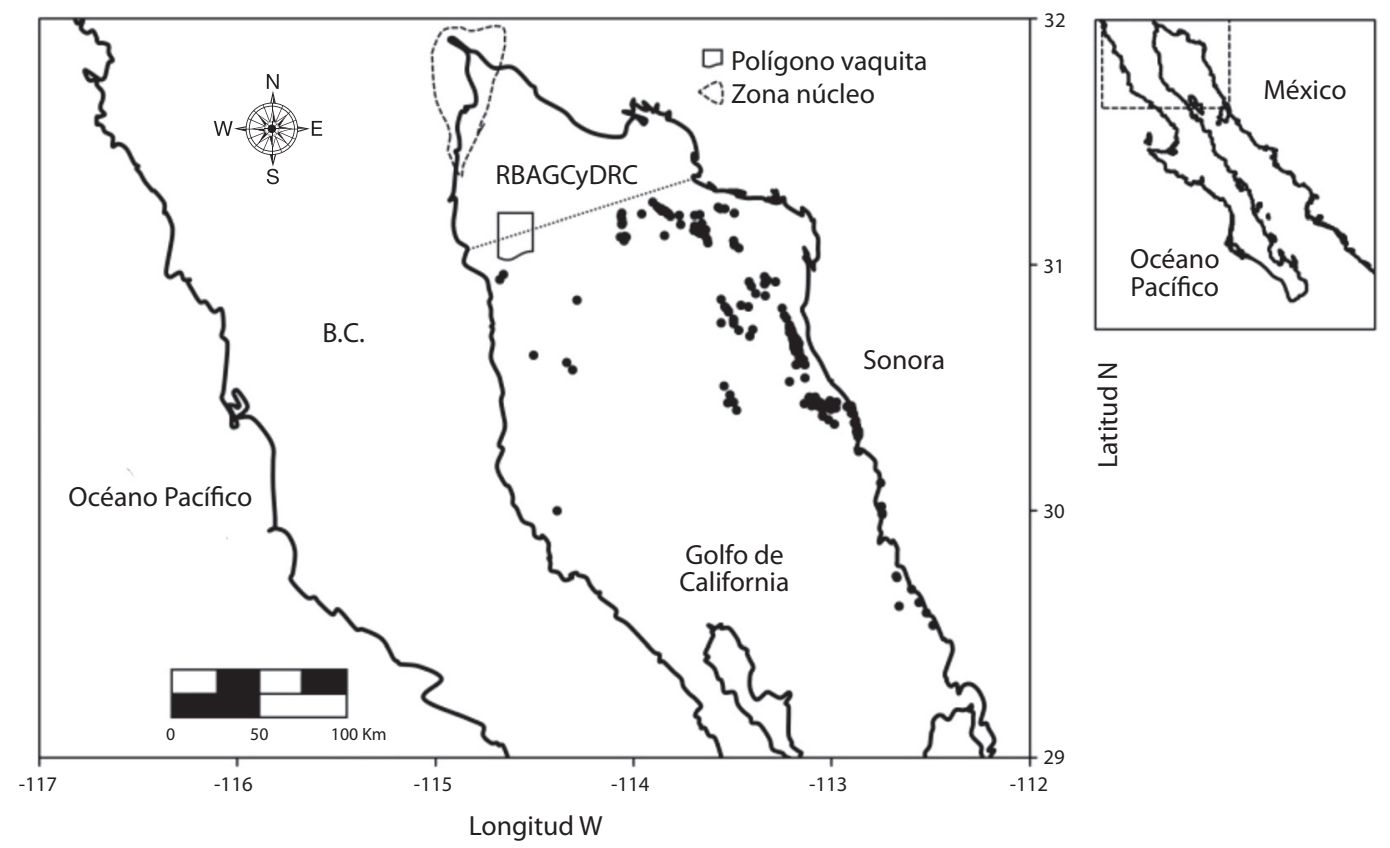

Fig. 1. Área de estudio en el Norte del Golfo de California, México.

Fig. 1. Study area in the Northern Gulf of California, Mexico.

y para Porichthys, Walker \& Rosenblatt (1988). Además, fue consultada la guía en línea de Robertson \& Allen (2015). Los nombres comunes fueron tomados de Love, Yoklavich \& Thorsteinson (2002) y Robertson \& Allen (2002, 2015). Se integró el listado sistemático de las especies para la construcción de la matriz de agregación taxonómica por lance y/o muestra analizada.

Para determinar el posible número de especies a encontrar en el ecosistema de forma mensual, se obtuvieron curvas de acumulación de especies utilizando los estimadores de riqueza no paramétricos Chao2, Jackknife 2 y Booststrap, aplicando 1000 iteraciones por modelo, todas las estimaciones se llevaron a cabo con PRIMER 6 (Clarke \& Gorley, 2006).

Diversidad taxonómica: Para identificar cambios en la estructura de la comunidad se utilizaron dos índices de diversidad taxonómica: El índice de distinción taxonómica promedio delta $\left(\Delta^{+}\right)$, que se basa en presencia/ ausencia de las especies y actúa como indicador cualitativo. Evalúa la riqueza junto con la distancia taxonómica entre cada par de especies, por medio de la matriz de agregación taxonómica previamente construida en niveles taxonómicos de filo, clase, orden, familia, género y especie, aplicando la nomenclatura y clasificación jerárquica propuesta por Nelson (2006).

Se define por la fórmula:

$$
\Delta^{+}=2 \frac{\sum \sum_{i<j} \omega_{i j}}{S(S-1)}
$$

donde $S$ es el número de especies en la muestra y $\omega_{\mathrm{ij}}$ es el peso taxonómico otorgado a la longitud de la rama que une a las especies $i$ y $j$ a través de un árbol taxonómico. Cada nivel recibe un valor discreto y proporcional dentro de una escala de 100 , así, $\omega_{\mathrm{ij}}=16.7$ para la misma especie, $33.3=$ especies diferentes en el mismo género, $50=$ misma familia pero diferente género, $66.7=$ mismo orden pero diferente familia, $83.3=$ misma clase pero diferente 
orden y $100=$ mismo filo pero diferente clase respectivamente (Palacios, 2011).

El índice de variación de la distinción taxonómica promedio o lambda $(\Lambda+)$, es matemáticamente la variancia de $\left(\Delta^{+}\right)$y mide como se encuentran representadas las especies (Clarke \& Warwick, 2001).

La matriz de agregación taxonómica general de las especies fue ordenada de especie a filo, a través de TAXDTEST en PRIMER V6. Esta rutina, realiza 1000 selecciones aleatorias de las especies generadas al azar del listado total de las especies presentes en el área, contrastando los valores de los índices (delta $\Delta+o$ lambda $\Lambda+$ ) y generando una distribución de probabilidad al $95 \%$ de confianza (Clarke \& Gorley, 2001; Clarke \& Warwick, 2001).

Con la finalidad de obtener los atributos ecológicos y caracteres morfológicos de cada especie presente en el listado sistemático, se revisaron bases de datos biológicas disponibles como www.fishbase.org, www.neotropicalfishes.org/step, Froese \& Pauly (2007), ITIS (2011) (Integrated Taxonomic Information System, on-line database http://www.itis.usda. gov/) y Robertson \& Allen (2015). Posteriormente, para obtener la diversidad funcional, se siguió el protocolo propuesto por Palacios (2011). Se consideraron los atributos ecológicos y caracteres morfológicos de cada especie en su etapa adulta (Mathieson et al., 2000; Tilman, 2001; Dumay, Tari, Tomasini, \& Mouillot, 2004; Gristina, Bahri, Fiorentino, \& Garofalo, 2006) relacionados con el funcionamiento del ecosistema (Somerfield, Clarke, Warwick, \& Dulvy, 2008).

Los caracteres usados para el análisis y la forma de agruparlos (Palacios 2011) fue la siguiente:

a. Nivel trófico (2-2.49, 2.5-2.99, 3-3.49, 3.5-3.99, 4-4.49, 4.50-4.99).

b. Gremio trófico: carnívoros de primer orden y se alimentan principalmente de invertebrados bentónicos; ictio-invertívoros que son carnívoros de segundo orden y se alimentan de invertebrados bentónicos e incluyen una proporción de peces;
Piscívoros cuya dieta está compuesta por peces e incluyen una proporción de invertebrados; planctívoros son consumidores del plancton; omnívoros peces que combinan las algas con invertebrados y detritus (Mejía \& Garzón-Ferreira, 2000).

c. Gremio reproductivo: Vivíparos (V), Ovovivíparos (W), Ovíparos (O), los últimos subdivididos en ovíparos con huevos pelágicos (OP), ovíparos con huevos bentónicos y fase pelágica (OBFP), ovíparos con huevos bentónicos sin fase pelágica (OBSFP), ovíparos con gestación oral (OGO) y ovíparos con gestación en bolsa ventral vascularizada (OGBVV).

d. Posición en la columna de agua: pelágiconeríticas $(\mathrm{PN})$; pelágico-oceánico-neríticas (PON); demerso-pelágicas (DP); demersal subdividida en especies asociadas a fondos blandos (DFB); especies asociadas a fondos mixtos (DFM); especies asociadas a fondos duros (DFD).

e. Grupo de tallas: peces óseos T-I $<29.9 \mathrm{~cm}$; T-II $\geq 30 \leq 49.9 \mathrm{~cm}$; T-III $\geq 50 \mathrm{~cm}$, elasmobranquios: T-IV $\geq 60 \mathrm{~cm}, \mathrm{~T}-\mathrm{V} \geq 105.1 \mathrm{~cm}$.

f. Forma del cuerpo: fusiforme, anguiliforme, compresiforme, compresiforme A, depresiforme, depresiforme A, depresiforme B, globiforme, sagitiforme, teniforme y otros.

g. Tipo de boca: oblicua, superior, terminal, inferior (incluye las de tipo semiventral), ventral, protráctil, tubular e incluida (Miller \& Lea, 1972; Barton, 2006, Palacios 2011).

Como medida de similitud se utilizó el índice de Bray-Curtis (1957), cuya matriz sirvió para llevar a cabo un análisis de agrupamiento para la formación de grupos funcionales, utilizando los rasgos de las especies. La hipótesis (Ho) de no diferencias en los atributos funcionales de la comunidad, fue evaluada a través del análisis de varianza de una vía, ANOSIM.

Para los análisis de contribución de las especies, se utilizó un análisis de similitud de porcentajes, a través de SIMPER, llevando a cabo el corte a un nivel de $90 \%$ de acumulación 
de la similitud (Clarke \& Warwick, 2001). Para comprobar diferencias en la composición de las especies entre grupos y por cruceros, se aplicó la prueba de análisis multifactorial de similitudes de una vía, con ANOSIM (Clarke \& Green 1988, Clarke \& Warwick, 2001). Si el valor de $\mathrm{R}$ es próximo a 1 , aumenta el grado de discriminación entre los grupos y se rechaza Ho. Si el valor de $\mathrm{R}$ es aproximadamente 0 , indica que en promedio la similitud entre grupos es idéntica y no se rechaza Ho. Pero si R es negativa se sugiere baja riqueza de especies en la comunidad, alta variabilidad en una muestra o se presume que fue incorrecto el acomodo de las muestras para comparar (Palacios, 2011).

Todos los análisis fueron realizados con el software PRIMER v6 (Clarke \& Gorley, 2006).

\section{RESULTADOS}

Composición de especies: Los peces representaron $70.7 \%$ del total de la captura, con 32757 individuos, pertenecientes a dos clases, 26 ordenes, 56 familias, 93 géneros y 148 especies. El orden Perciformes fue el más diverso con 18 familias, 25 géneros y 43 especies. Las familias mejor representadas fueron: Sciaenidae (21), Paralichthydae (11),
Haemulidae y Serranidae (10 cada uno). Los géneros mejor representados en número de especies son: Symphurus (cinco), Diplectrum y Cynoscion (cuatro).

La curva de acumulación de especies con los estimadores de Chao2, Jacknife2 y Bootstrap no mostró la asíntota (Fig. 2). El estimador Bootstrap fue el más estabilizado, con $92 \%$ de aceptación en el número de especies observadas, con valores de 189 y 160 especies, respectivamente.

\section{El índice de distinción taxonómica pro-} medio $(\Delta+)$ : Para las temporadas de pesca 2009-2010 y 2010-2011, este índice mostró lances que se ubicaron sobre y debajo de la media de la distribución de probabilidad $95 \%$ y cinco lances por fuera del límite inferior de la distribución. Por otro lado, el valor máximo de la distancia en el árbol taxonómico, fue en el lance $36=76.37$ en septiembre $(2010-2011)$ y la menor distancia en el lance $18=50.00 \mathrm{del}$ mes de agosto (2011) (Fig. 3a). Por su parte, el índice de variación de la distinción taxonómica promedio $(\Lambda+)$, siguió el mismo comportamiento, la mayoría de los lances se distribuyen por encima y por debajo de la media; cuatro lances correspondientes a enero y marzo

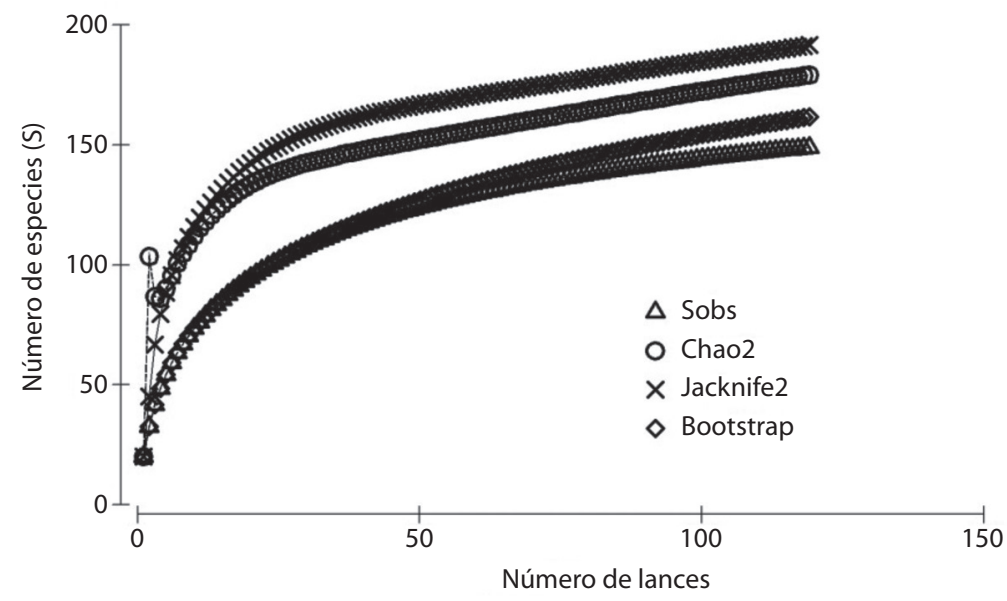

Fig. 2. Curva de acumulación de especies con los estimadores Chao2, Jacknife2, Bootstrap y Sobs en las muestras obtenidas a bordo de barcos comerciales en el NGC, durante 2009-2010 y 2010-2011.

Fig. 2. Species accumulation curve with Chao2, Jacknife2, Bootstrap and Sobs in the samples obtained on board the commercial boats in the Northern Gulf of California during 2009-2010 and 2010-2011. 

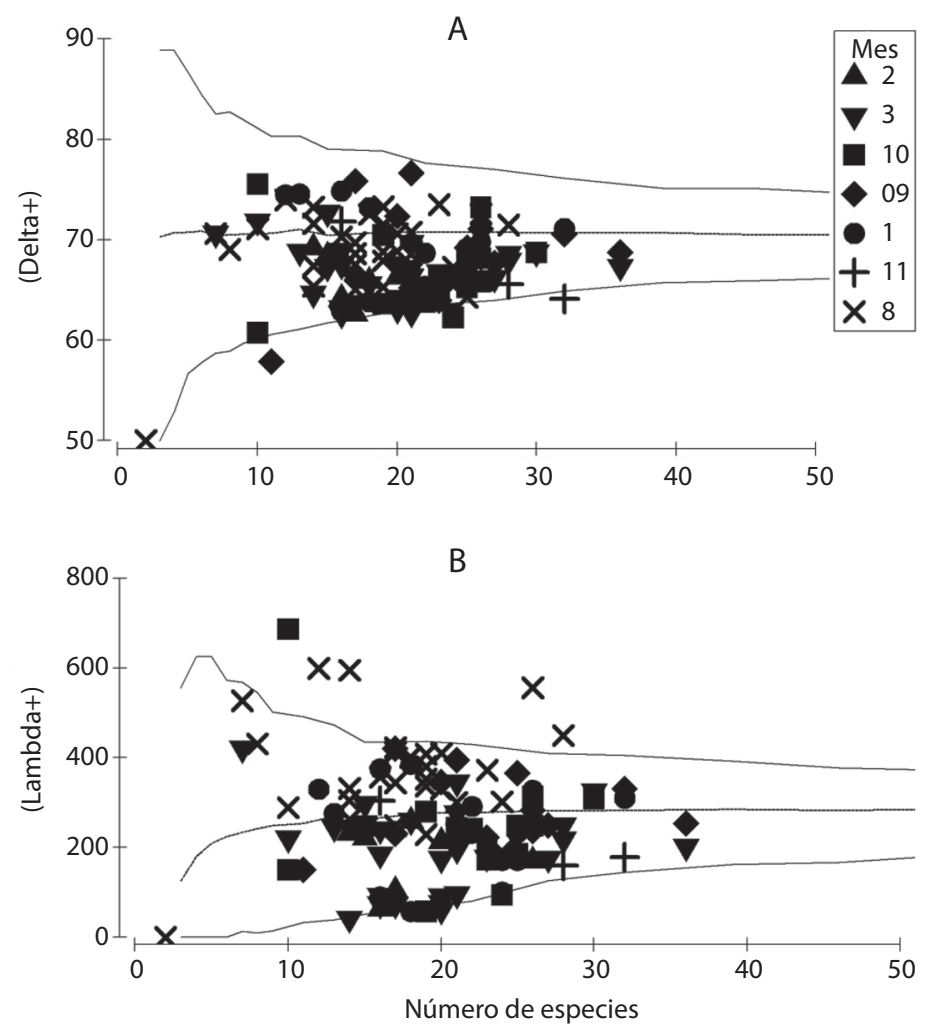

Fig. 3. Índice de distinción taxonómica promedio delta $+(\Delta+)$ e Índice de variación taxonómica promedio lambda $+(\Lambda+)$ en peces componente de la FAC, durante 2009-2010 y 2010-2011 en el NGC. Se muestra el promedio (línea central) y la distribución de probabilidad al $95 \%$ (líneas continuas).

Fig. 3. Average taxonomic distinction index delta $+\left(\Delta^{+}\right)$and average taxonomic variation index lambda $+(\Lambda+)$ in by-catch fish during 2009-2010 and 2010-2011 in the Northern Gulf of California. Central line shows average and continuous lines show distribution probability at $95 \%$.

cayeron en el límite de la distribución de probabilidad de $95 \%$. En tanto, cuatro lances de agosto 2011 y un lance de octubre, se ubicaron por fuera del límite superior de la distribución de probabilidad, lo que sugiere que los valores en la distinción taxonómica son mayores a la distinción estimada (Fig. 3b). Esto mismo se confirmó a través de un ordenamiento multidimensional no paramétrico nMDS con grado de estrés 0.21 (Fig. 4), que reflejó las diferencias en las distancias taxonómicas entre los lances de octubre y agosto, mismos que quedaron separados del resto. En los análisis de varianza de los índices, la distinción taxonómica promedio $\Delta+$ no mostró diferencias significativas $\mathrm{t}=2.447 ; \mathrm{p}=0.294$, mientras que para el índice de variación taxonómica promedio $\Lambda+$ si se encontraron diferencias estadísticamente significativas $\mathrm{t}=3.35 ; \mathrm{p}=0.00008$.

En los análisis multifactoriales de una vía ANOSIM por pares entre los grupos de cruceros (meses), la mayor afinidad se observó entre febrero-marzo $(\mathrm{R}=-0.092)$, mientras que los más disimilares fueron febrero-noviembre $(\mathrm{R}=$ 0.902) (Cuadro 1).

El análisis de similitud ANOSIM mostró una $\mathrm{R}=0.812$ y se formaron 17 grupos funcionales GF (Cuadro 2), donde los grupos (m) y (g) aportaron el $50 \%$ del total de las especies, mientras que los GF (j) y (o) presentaron solo una especie Bollmannia stigmatura e Hippocampus ingens. En los análisis de contribución 


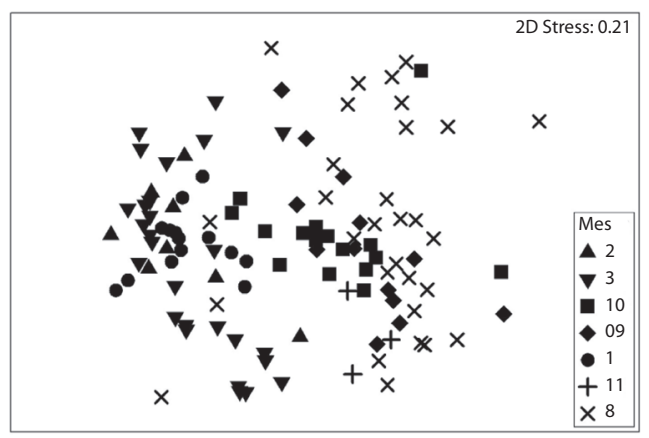

Fig. 4. Ordenamiento multidimensional no paramétrico nMDS para los lances analizados, con grado de estrés 0.21 basados en las distancias taxonómicas, en el NGC durante 2009-2010 y 2010-2011.

Fig. 4. Non-parametric multidimensional ordering nMDS for the throws analyzed with a degree of stress of 0.21 based on taxonomic distances in the Northern Gulf of California during 2009-2010 and 2010-2011.

promedio (SIMPER) entre rasgos funcionales dentro de la estructura de la comunidad, el GF (m) formado por 33 especies principalmente pequeñas y medianas, presentó $62.83 \%$ de similitud promedio SP en 7 rasgos funcionales (Cuadro 2). Entre las más abundantes y con mayor frecuencia de aparición se encontraron: Symphurus chabanaudi (65 lances), O. reddingi (58 lances), P. grandisquamis (54 lances), $P$. stephanophrys (51 lances), Citharichthys fragilis (52 lances) y E. crossotus (47 lances). El GF (g) estuvo integrado por 38 especies con SP de $57.43 \%, 8$ de las especies más abundantes y mayor aparición fueron: Synodus scituliceps (75 lances), Diplectrum macropoma (72 lances), Micropogonias megalops (68 lances) y Paralabrax maculatofasciatus (28 lances). El GF (i) presentó una SP de $51.19 \%$, con especies pelágicas-neríticas, de huevo ovíparo pelágico y tallas pequeñas (Cuadro 2). Las especies del GF (k) presentaron SP $72.87 \%$, siendo $B$. polylepis la especie más frecuente $(52$ lances). El GF (e) presentó una SP de $63.47 \%$, con especies de tamaño mediano $(\geq 30 \leq 49.9$ $\mathrm{cm})$ y demerso-pelágicas, siendo $P$. panamensis la especie más importante tanto en abundancia, como en frecuencia de aparición (82 lances). El GF (f) presentó una SP de $63.37 \%$, integrado por 19 especies de formas y tallas variables,
CUADRO 1

Prueba de similitud entre cruceros (meses)

TABLE 1

Similarity test between exploration cruises (months)

\begin{tabular}{ccc} 
Among groups & Statistical R & $\begin{array}{c}\text { Significance } \\
\text { level }(\%)\end{array}$ \\
2.3 & -0.092 & 85.9 \\
2.10 & 0.588 & 0.1 \\
2.9 & 0.369 & 0.3 \\
2.1 & 0.561 & 0.1 \\
2.11 & 0.902 & 0.6 \\
2.8 & 0.418 & 0.1 \\
3.10 & 0.517 & 0.1 \\
3.9 & 0.625 & 0.1 \\
3.1 & 0.354 & 0.1 \\
3.11 & 0.595 & 0.1 \\
3.8 & 0.550 & 0.1 \\
10.9 & 0.088 & 0.8 \\
10.1 & 0.496 & 0.1 \\
10.11 & 0.259 & 13.7 \\
10.8 & 0.140 & 2.6 \\
9.1 & 0.481 & 0.1 \\
9.11 & 0.085 & 32.8 \\
9.8 & 0.209 & 0.5 \\
1.11 & 0.726 & 0.2 \\
1.8 & 0.443 & 0.1 \\
11.8 & 0.203 & 14.4 \\
\hline & &
\end{tabular}

$\mathrm{R}=0.364$ basada en el Coeficiente de similitud de BrayCurtis, en el NGC durante 2009-2010 y 2010-2011.

$\mathrm{R}=0.364$ based on Bray-Curtis similarity coefficient in the Northern Gulf of California during 2009-2010 and 2010-2011.

con cuerpo anguiliforme $\geq 50 \mathrm{~cm}$ y cuerpo depresiforme B y depresiforme A con tallas $>$ $29 \mathrm{~cm}$, todas de huevo ovíparo pelágico.

El GF (j) estuvo integrado por una sola especie $B$. stigmatura, por lo tanto una SP de $100 \%$, especie carnívoro primario de talla $>29$ $\mathrm{cm}$ y nivel trófico de 3.3. El GF (p) con una SP de $64.11 \%$ integrado por especies cartilaginosas principalmente, Dasyatis diapterura y D. longa. El GF (d) con solo tres especies que comparten el $80.95 \%$ de la SP y difieren únicamente en la boca y tipo de cuerpo. El GF (b) con una SP de $66.67 \%$ integrado por los tiburones Galeorhinus galeus y Heterodontus 
CUADRO 2

Grupos funcionales de acuerdo a rasgos funcionales ecológicos y de morfología en FAC en el NGC durante 2009-2010 y 2010-2011

TABLE 2

Functional groups according to the ecological and morphological functional traits in by-catch in the Northern Gulf of California during 2009-2010 y 2010-2011

$\begin{array}{lcc}\text { ESPECIES / SM=similitud promedio SIMPER } & \text { Atributo } & \text { Contrib.\% } \\ \text { GM Achirus mazatlanus, Bairdiella icistia, Bellator xenisma, Cheilotrema, fasciatum, Citharichthys } & \text { R-OP } & 22.75 \\ \text { platophrys, Citharichthys fragilis, Citharichthys gilberti, Etropus crossotus, Eucinostomus } & \text { A-ZBF } & 20.04 \\ \text { currani, Eucinostomus dowii, Eucinostomus entomelas, Eucinostomus gracilis, Haemulon } & \text { C-DFB } & 17.5 \\ \text { steindachneri, Haemulopsis elongatus, Haemulopsis nitidus, Halichoeres semicinctus, } & \text { NT-3 } & 16.29 \\ \text { Menticirrhus elongatus, Menticirrhus nasus, Menticirrhus panamensis, Menticirrhus undulatus, } & \text { T-I } & 5.17 \\ \text { Micropogonias ectenes, Orthopristis reddingi, Pleuronichthys guttulatus, Pleuronichthys ocellatus, } & \text { B-I } & 5.17 \\ \text { Prionotus albirostris, Prionotus birostratus, Prionotus horrens, Prionotus ruscarius, Prionotus } & \text { T-II } & 3.92\end{array}$
stephanophrys,Pseudupeneus grandisquamis,Symphurus chabanaudi, Symphurus fasciolaris, symphurus gorgonae, Symphurus leei, Xystreurys liolepis, Zalieutes elater.

\section{SP $62.83 \%$}

GG Albula esuncula, Albula pacifica, Ancylopsetta dendritica, Caranx caballus, Caranx vinctus, , Cynoscion othonopterus, Cynoscion parvipinnis, Cynoscion reticulatus, Cynoscion squamipinnis, Cynoscion stolzmanni, Cynoscion xanthulus, Diplectrum eumelum, Diplectrum labarum, Diplectrum macropoma, Diplectrum pacificum, Diplectrum sciurus, Haemulon scudderi, Hippoglossina tetrophthalma, Hyporthodus acanthistius, Hyporthodus niphobles, Isopisthus remifer, Lutjanus guttatus, Micropogonias altipinnis, Micropogonias megalops, Paralabrax maculatofasciatus, Paralichthys aestuarius, Paralichthys californicus, Paralichthys woolmani, Rypticus nigripinnis,

NT-4 $\quad 2.76$

\section{SP $57.43 \%$}

Gi Anchoa ischana, Anchoa nasus, Caranx otrynter, Cetengraulis mysticetus, Fodiator acutus, Opisthonema libertate, Peprilus ovatus, Peprilus snyderi, Sardinops sagax, Physiculus nematopus, Scomber japonicus.

$\begin{array}{cc}\text { R-OP } & 25.63 \\ \text { A-ZPF } & 21.4 \\ \text { C-PN } & 21.4 \\ \text { NT-3 } & 8.12 \\ \text { C-F } & 7.95 \\ \text { T-I } & 5.82 \\ \text { T-II } & \\ \text { NT-3 } & 20.63 \\ \text { R-OP } & 20.63 \\ \text { B-T } & 13.89 \\ \text { T-III } & 13.47 \\ \text { C-C } & 13.47 \\ \text { C-DFM } & 8.46 \\ \text { T-II } & 23.39 \\ \text { NT-4 } & 23.39 \\ \text { R-OP } & 23.39 \\ \text { C-DP } & 12.69 \\ \text { A-ICBF } & 8.04 \\ \text { A-ICBF } & 22.34 \\ \text { R-OP } & 22.34 \\ \text { C-DFB } & 19.35 \\ \text { NT-4 } & 9.66 \\ \text { C-A } & 9.66 \\ \text { B-SV } & 6.01 \\ \text { T-I } & 3.22 \\ & \end{array}$




\begin{tabular}{|c|c|c|c|}
\hline & ESPECIES / SM=similitud promedio SIMPER & Atributo & Contrib.\% \\
\hline GJ & $\begin{array}{l}\text { Bollmannia stigmatura } \\
\text { SP } 100 \% \text { Características únicas }\end{array}$ & & 100 \\
\hline GP & $\begin{array}{l}\text { Dasyatis dipterura, Dasyatis longa, Gymura marmorata, Myliobatis californica, Narcine } \\
\text { entemedor, Raja equatorialis, Raja velezi, Rhinobatos productos, Urobatis halleri, Urobatis } \\
\text { maculatus, Urotrygon chilensis, Urotrygon rogersi, Zapteryx exasperata. } \\
\text { SP } 64.11 \%\end{array}$ & $\begin{array}{l}\text { B-V } \\
\text { C-DA } \\
\text { R-V } \\
\text { A-ICBF } \\
\text { C-DFM } \\
\text { T-V } \\
\text { NT-3 }\end{array}$ & $\begin{array}{r}22.65 \\
22.65 \\
18.95 \\
12.71 \\
5.89 \\
4.64 \\
4.42\end{array}$ \\
\hline GD & $\begin{array}{l}\text { Fistularia commersonii, Fistularia corneta, Selene peruviana. } \\
\text { SP } 80.85 \%\end{array}$ & $\begin{array}{l}\text { T-III } \\
\text { C-DP } \\
\text { A-ICF } \\
\text { NT-5 } \\
\text { R-OP } \\
\text { B-T1 }\end{array}$ & $\begin{array}{r}17.65 \\
17.65 \\
17.65 \\
17.65 \\
17.65 \\
5.88\end{array}$ \\
\hline GB & $\begin{array}{l}\text { Galeorhinuss galeus Heterodontus francisci. } \\
\text { SP } 66.67 \%\end{array}$ & $\begin{array}{l}\text { T-IV } \\
\text { B-I } \\
\text { A-ICBF } \\
\text { R-OB } \\
\text { C-D }\end{array}$ & $\begin{array}{l}20 \\
20 \\
20 \\
20 \\
20\end{array}$ \\
\hline $\mathrm{GH}$ & $\begin{array}{l}\text { Haemulon maculicauda, Hemanthias pernanus Larimus acclivis, Larimus effulgens, Larimus } \\
\text { pacificus, Umbrina xanti, Xenistius californiensis. } \\
\text { SP } 69.28 \%\end{array}$ & $\begin{array}{l}\text { C-DP } \\
\text { C-F } \\
\text { NT-3 } \\
\text { R-OP } \\
\text { A-ZPF } \\
\text { T-II }\end{array}$ & $\begin{array}{c}21.07 \\
21.07 \\
15.11 \\
14.73 \\
10.12 \\
9.82\end{array}$ \\
\hline GO & Hipocampus ingens Características únicas SP 100 \% & & 100 \\
\hline GN & $\begin{array}{l}\text { Mustelus californicus, Mustelus lunulatus. } \\
\text { SP } 66.67 \%\end{array}$ & $\begin{array}{l}\text { B-V } \\
\text { C-D } \\
\text { R-V } \\
\text { NT-4 } \\
\text { A-ICBF }\end{array}$ & $\begin{array}{l}20 \\
20 \\
20 \\
20 \\
20\end{array}$ \\
\hline $\mathrm{GC}$ & $\begin{array}{l}\text { Occidentarius platypogon, Opistognathus punctatus, Arius sp. } \\
\text { SP } 71.43 \%\end{array}$ & $\begin{array}{l}\text { B-I } \\
\text { A-ICBF } \\
\text { R-OGO } \\
\text { C-DB } \\
\text { T-II } \\
\text { C-DFB }\end{array}$ & $\begin{array}{c}20 \\
20 \\
20 \\
20 \\
6.67 \\
6.67\end{array}$ \\
\hline GA & $\begin{array}{l}\text { Porichthys analis, Porichthys mimeticus } \\
\text { SP } 71.73 \%\end{array}$ & $\begin{array}{l}\text { C-DFB } \\
\text { B-O } \\
\text { A-ABF } \\
\text { R-OBSP } \\
\text { C-DB }\end{array}$ & $\begin{array}{l}20 \\
20 \\
20 \\
20 \\
20\end{array}$ \\
\hline GI & Sphoeroides annulatus, Sphoeroides sechurae & $\begin{array}{l}\text { B-I } \\
\text { A-ABF } \\
\text { R-OBFP } \\
\text { R-OP } \\
\text { C-G }\end{array}$ & $\begin{array}{l}20 \\
20 \\
20 \\
20 \\
20\end{array}$ \\
\hline
\end{tabular}


francisci con diferente nivel trófico: G. galeus es un depredador primario y $\mathrm{H}$. francisci es un consumidor primario. El GF (h) con el $69.28 \%$ de SP y cinco especies de la familia Sciaenidae, dentro de estas L. effulgens, registrada en las coordenadas $\left(114^{\circ} 20^{\prime} 13.44^{\prime}\right.$ ' W - 30³6'11.99" $\mathrm{N})$. El GF (o) estuvo integrado por $H$. ingens con una SP de $100 \%$. El GF (n) integrado por Mustelus sp. con una SP de $100 \%$.

En tanto, el GF (c) estuvo integrado por tres especies con una SP de $71.43 \%$; GF (a) integrado por dos especies con una SP de $71.43 \%$ de las cuales Porichthys analis contribuyó con mayor biomasa y abundancia. El GF (i) también con dos especies y una SP de $72.50 \%$.

La diversidad funcional en los atributos ecológicos mostró una comunidad integrada por especies con nivel trófico intermedio a alto: $49.3 \%$ de nivel 3-3.4 (carnívoros primarios), $30.4 \%$ nivel 3.5-3.9 (carnívoros secundarios), entre otros (Fig. 5a), formando parte de diferentes grupos funcionales.

Los grupos de tallas grandes $(6.7 \%)$ fueron representados por tiburones y rayas de longitud total $\geq 30 \leq 49.9 \mathrm{~cm}$, mientras el $25 \%$ lo representaron las tallas $<29 \mathrm{~cm}$ (Fig. $5 b$ ). En el gremio reproductivo, la dominancia de organismos ovíparos con huevos con fase pelágica OP fue alta $(83.8 \%)$ y un pequeño porcentaje (9.5 $\%)$ lo explicó la reproducción vivípara $\mathrm{V}$ de los tiburones y rayas, el resto estuvo representado por las otras formas de reproducción (Fig. 5c). En la distribución en la columna de agua 48.6 $\%$ de las especies fueron demersales con preferencia a fondos blandos (DFB), $23 \%$ fueron dermersales-pelágicas y el $14.5 \%$ de las especies demersales con preferencia a los fondos mixtos (DFM), en tanto $11.5 \%$ fueron especies pelágicas-neríticas y hubo escasa presencia (1 $\%$ ) de especies con preferencia a fondos duros (Fig. 5d). En el gremio alimenticio, la comunidad estuvo integrada por ictiobentófagos (52 $\%$ ), zoobentófagos (30\%), zooplanctófagos (11 $\%$ ), ictiófagos (3.4\%) y omnívoros (2\%) (Fig. $5 \mathrm{e})$. De acuerdo a la forma del cuerpo, la comunidad se integró por peces fusiformes $(38.5 \%)$, depresiforme A (21.5\%), depresiforme B (11.5
\%) y anguiliforme y compresiforme (Fig. 5f). En cuanto a tipo y posición de la boca, la boca inferior fue la más frecuente $(20.3 \%)$, seguida de la terminal (17.6\%), la proyectante (16.9 $\%)$ y finalmente de la boca en posición superior (12\%) (Fig. 5g).

\section{DISCUSIÓN}

La parte norte del Golfo de California (NGC) se ha considerado como una zona mega diversa de alto endemismo (López, Herrera, Rodríguez, \& Hernández, 2010); a su vez, presenta gran diversidad taxonómica que incluyen especies de peces tropicales, subtropicales, templadas, ártico boreales o templado cálidas como resultado del potencial alimenticio autotrófico, sumado a la importancia de especies endémicas como Micropogonias megalops y Totoaba macdonaldi.

Esta parte del golfo, pertenece al Pacifico Oriental Tropical (POT) debido a que incluye las provincias biogeográficas de Cortez, Panámica y Mexicana. La Provincia de Cortez incluye todo el Golfo de California, hasta Topolobampo, Sinaloa (Hastings, 2000). Para la misma área de estudio, Palacios (2011) reportó 17 órdenes, 45 familias, 63 géneros y 73 especies, con la mayor riqueza de especies en niveles tróficos altos y organismos de tallas grandes, especialmente tiburones y rayas, siendo los teleósteos pequeños un pequeño porcentaje.

La mayor presencia de tiburones como componentes de la Fauna Acompañante reportada por Palacios (2011) en comparación con este estudio, puede ser explicada por el hecho de que en ese periodo (1993-1994) los elasmobranquios eran sujetos de pesca por embarcaciones menores, embarcaciones mayores dedicadas a la pesca de escama y por la misma flota camaronera, sugiriendo una potencial baja en la abundancia de los elasmobranquios en la zona, hecho que deberá corroborarse con estudios dedicados específicamente a evaluar cambios en la abundancia de los elasmobranquios en la región. En este trabajo los peces 

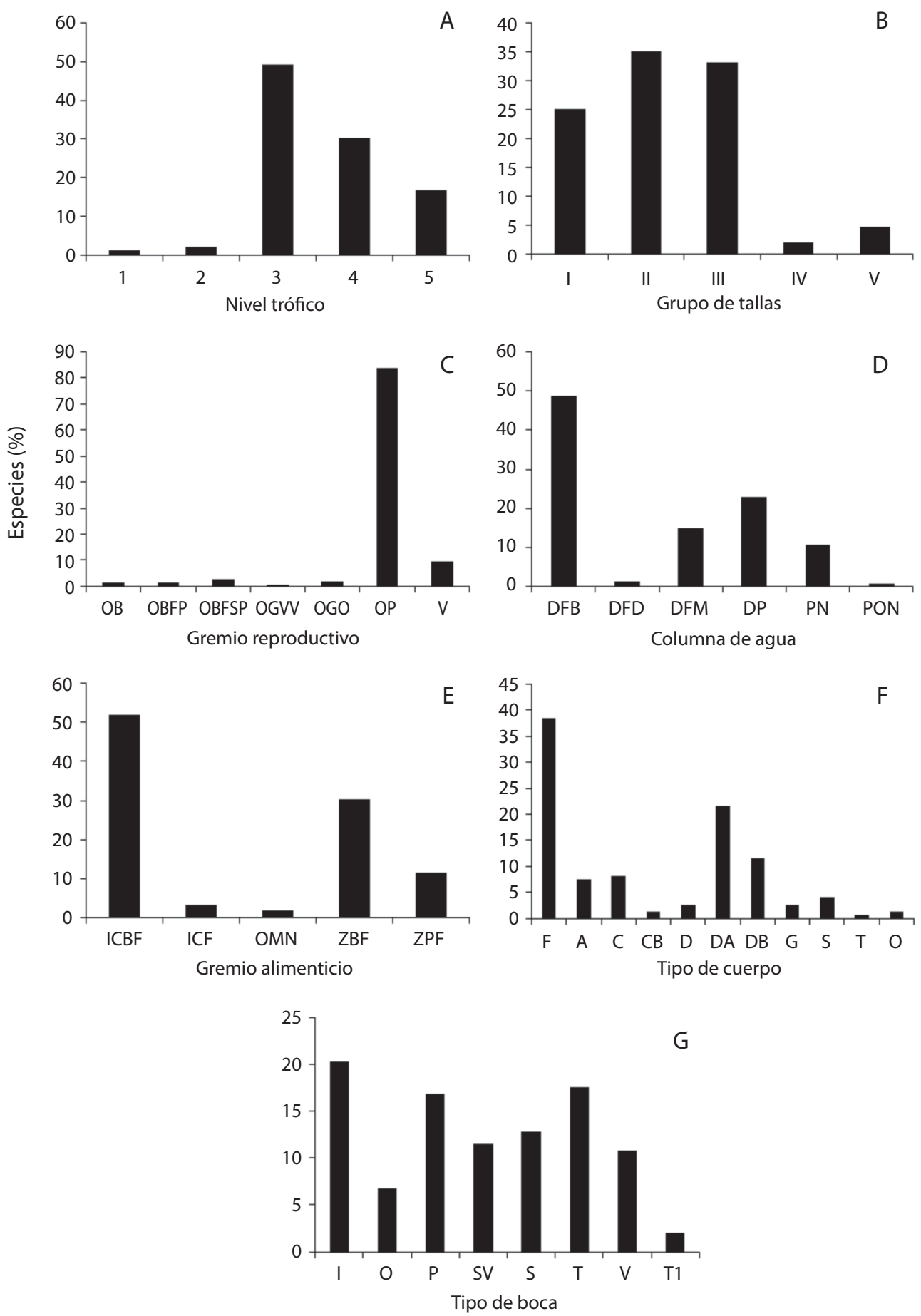

Fig. 5. Estructura de la comunidad basados en atributos ecológicos y rasgos morfológicos componentes de la FAC durante 2009-2010 y 2010-2011 en el norte del Golfo de California.

Fig. 5. Community structure based on ecological attributes and morphological traits of by-catch during 2009-2010 and 2010-2011 in the Northern Gulf of California. 
teleósteos dominaron los niveles tróficos altos dentro de la comunidad.

Especies como P. analis, S. scituliceps, Scorpaena sonorae, U. halleri, P. panamensis y D. macropoma fueron registradas como importantes tanto en biomasa, como en frecuencia de presencia en 1994 (Nava, 1994), en 2011 (Calderón, 2011) y en este estudio. Dichas especies son de amplia distribución latitudinal y batimétrica, y pertenecen al Pacífico Oriental Tropical (POT) (Robertson \& Allen, 2002, Robertson \& Allen, 2015). Por otra parte, especies restringidas al NGC como Cynoscion othonopterus y Totoaba macdonaldi raramente aparecieron.

Las especies P. analis, S. scituliceps, Scorpaena sonorae, $U$. halleri, $P$. panamensis y $D$. macropoma forman parte de las 30 especies más abundantes dentro de la FAC a lo largo del Golfo de California (Pérez \& Findley 1985; 88; López, Hernández, Herrera, Rábago, \& Morales, 2007; Madrid, Amezcua, \& Morales, 2007; Rábago et al., 2010, 2011), incluyendo el NGC (Nava, 1994; Calderón, 2011), donde la ictiofauna pertenece en su mayoría al POT (Castro, Balart, \& Arvizu. 1995).

La amplia distribución de estas especies se debe a su adaptación biológica a diversos hábitats y a las condiciones ambientales en la zona costera (López et al., 2010), considerada como un ambiente natural altamente dinámico y cambiante. Además, el NGC es considerado como uno de los ecosistemas marinos más ricos del mundo (Cudney \& Turk, 1998), lo que hace posible sostener grandes cadenas alimenticias, hecho que se refleja en la composición de los grupos funcionales de la comunidad de peces que forman parte del ecosistema. Cabe mencionar que en los registros de los autores mencionados y en este documento se identifican las especies en las cuales la pesca de arrastre está incidiendo de manera importante, en profundidades entre 25-40 m; sin embargo, es probable que varias de las especies tengan una distribución batimétrica mayor (Rábago et al., 2011) y se ha mencionado que solamente una porción de la población de esas especies está sujeta a presión pesquera (López et al., 2012). Entonces, debido a la misma dinámica del NGC y a los procesos naturales anteriormente mencionados, es de esperar cambios temporales en la disponibilidad de las especies, lo que explicaría los cambios mensuales en las contribuciones de algunas especies como $P$. analis y $S$. scituliceps, $U$. halleri, $S$. sonorae, D. macropoma, $P$. panamensis y $M$. megalops.

En estudios recientes se han adicionado los índices de distinción taxonómica promedio delta + y el índice de variación taxonómica promedio lambda + (Clarke \& Warwick, 2001), considerados amigables para los estudios de diversidad taxonómica y funcional de los ecosistemas para registrar cambios relevantes en las comunidades de peces (Ramos-Miranda et al., 2005), ya que no dependen del tamaño de muestra ni del esfuerzo del muestreo, y solamente utilizan atributos y caracteres ecológicos de las especies y con ello permiten identificar las características claves asociadas con la función en el ecosistema, siendo la estructura taxonómica de una comunidad una faceta importante de la biodiversidad (Mathieson et al., 2000; Dumay et al., 2004; Gristina et al., 2006). Mucho depende del grado de alteración que sufre una comunidad para que las alteraciones dentro de esta se puedan evidenciar. $\mathrm{Si}$ el efecto de la alteración es muy grande (sea de origen antropogénico o natural), la comunidad quizá experimente cambios taxonómicos no detectados con otras herramientas de estudio como los predictores ecológicos clásicos de Shannon-Wiener (1949) y Simpson (1949), y es por ello que la utilización de los índices de variación taxonómica son de gran ayuda (Clarke \& Warwick, 2001), ya que valores bajos en los índices de diversidad taxonómica son indicativos de un sistema alterado (Gristina et al., 2006). Sin embargo, en algunos estudios donde los índices de diversidad taxonómica han sido usados para evaluar cambios generados por alteraciones antropogénicas, no han sido lo suficientemente robustos (Aldea, Rosenfeld, \& Cárdenas, 2011).

En general, los análisis aquí reportados mostraron una comunidad bien estructurada, con diferencias taxonómicas por la gran variedad de formas y funciones dentro de la 
comunidad (Ramos-Miranda et al., 2005) y por la alta diversidad taxonómica en su conjunto, coincidiendo con lo mencionado por Palacios (2011). Otros estudios mencionan que las variaciones en la distinción taxonómica promedio en las comunidades analizadas se deben a las variaciones ambientales más que al esfuerzo pesquero (Nieto, 2010; Escobar, 2012; Nieto et al., 2013).

Aún cuando se observaron lances por debajo del límite de distribución de probabilidad esperada $95 \%$ de acuerdo al índice de distinción taxonómica promedio delta + , y lances por arriba y abajo del límite superior e inferior del contorno de probabilidad del $95 \%$ en el índice de variación taxonómica promedio lambda+, estos resultados no indicaron cambios significativos en la distancia taxonómica, debido a que el $96 \%$ del total de lances se encontraron dentro del estimado de probabilidad esperada.

Tilman (2001), plantea que se puede generalizar la diversidad funcional como el conjunto de caracteres de los organismos que influyen en las propiedades del ecosistema y que un ecosistema con alta diversidad funcional opera más eficientemente en términos de productividad, resiliencia, resistencia; siendo la resiliencia el indicador más general del funcionamiento del ecosistema, debido a la capacidad de resistir o recuperarse ante una alteración. Por lo que, el número de especies por GF encontradas en este estudio, muestra un ecosistema sano de acuerdo con Naeem (1994) y Hooper et al. (2005), que mencionan que entre más especies tenga un GF será mejor para el ecosistema.

Al poseer los GF alta diversidad taxonómica, se reduce la probabilidad de que ingresen especies exóticas para reemplazar las existentes (Naeem, 1994), por lo que entre más especies dominantes o subordinadas se agrupen, se incrementará la redundancia funcional y por ende aumentará la resiliencia del ecosistema para responder a posibles alteraciones de origen natural o antropogénico (Palacios, 2011), ya que habrá más especies que realicen la misma función. Caso contrario, los GF integrados por pocas especies como el caso de tiburones, corren el riesgo de verse afectados con la pérdida de una sola especie. El caso extremo es el de los grupos formados por una sola especie, como el del caballito de mar $H$. ingens y $B$. stigmatura, (enlistado el primero en UICN 2014 como especie vulnerable (http:// www.iucnredlist.org/details/10072/0), donde la perdida de la especie implicaría la desaparición de ese grupo funcional.

Manejar e interpretar grupos funcionales es posible pero altamente complejo y su uso poco frecuente en estudios ecosistémicos (Brusca \& Findley, 2005), sin embargo, es altamente recomendable el estudio de los aspectos ecológicos, taxonómicos y funcionales de las especies de peces asociadas a la pesca de arrastre en diversas partes del mundo.

\section{AGRADECIMIENTOS}

Esta investigación fue financiada con el proyecto CONACYT 215355, se agradece al Centro de Investigaciones Biológicas del Noroeste, S.C., en particular a los proyectos EP1 y Produce Sonora 897-1 y a Rufino Morales del laboratorio de Pesquerías de la Unidad Guaymas del CIBNOR. La edición en inglés fue revisada por Diana Dorantes.

\section{RESUMEN}

La parte norte del Golfo de California es una zona mega diversa de alto endemismo con gran interés económico por las pesquerías multiespecíficas que se desarrollan, principalmente de camarón. Existe carencia de estudios recientes sobre ensamblajes de peces componentes de la fauna acompañante. Por lo que, durante las temporadas de pesca $2009-2010$ y 2010-2011 se realizaron 14 viajes de pesca comercial a bordo de 13 barcos camaroneros, con un total de 119 lances, efectuados entre los 5 y $90 \mathrm{~m}$ de profundidad. Los 119 lances fueron analizados para evaluar la estructura de la comunidad de peces. Se utilizaron índices de diversidad taxonómica para detectar cambios taxonómicos en la comunidad siguiendo el índice de distinción taxonómica promedio $\Delta^{+} \mathrm{y}$ el índice de variación taxonómica $\Delta^{*}$ (TAXDEST del programa PRIMER v6). Para la conformación de grupos funcionales se consideró las similitudes de rasgos ecológicos y morfológicos entre las especies. Los resultados mostraron que los índices $\Delta^{+} \mathrm{y}$ $\Delta^{*}$ estuvieron dentro del promedio estimado y por dentro de los intervalos de confianza al $95 \%$, se encontraron 
diferencias significativas entre los índices. Los análisis mostraron una comunidad bien estructurada debido a la gran variedad de formas y funciones de las especies dentro de la comunidad. En la composición de grupos funcionales, el atributo ecológico que más contribuyó para conformación grupos, fue el gremio reproductivo. La estructura de la comunidad fue representada por niveles tróficos intermedios 3-3.9, preferencialmente carnívoros primarios y carnívoros secundarios, dentro de las categorías tróficas de ictiobentófagos y zoobentófagos, pertenecientes a especies demersales de fondos blandos y mayormente de cuerpo fusiforme.

Se concluye que el NGC presentó alta redundancia funcional de acuerdo a los grupos funcionales estimados, por lo que se considera un ecosistema estable y de gran diversidad. Es recomendable dar seguimiento a este tipo de estudios utilizando información de esfuerzo pesquero y ambiental, debido a la gran importancia biológica y ecológica del área.

Palabras clave: peces, Norte Golfo de California, diversidad funcional, diversidad taxonómica.

\section{REFERENCIAS}

Aldea, C., Rosenfeld, S. \& Cárdenas, J. (2011). Caracterización de la diversidad de moluscos bentónicos sublitorales en isla Carlos III y áreas adyacentes, estrecho de Magallanes, Chile. Anales Instituto Patagonia, 39(2), 73-89.

Barton, M. (2006). Bond's biology of fishes. 3er Edition, Thomson Brooks/Cole.

Box, E. G., Stuart, J. H., \& Hunter, W. G. (2008). Estadística para investigadores. Diseño, innovación y descubrimiento. España: Wiley, Barcelona.

Bray, J. R., \& Curtis, J. T. (1957). An ordination of the upland forest communities of Southern Wisconsin. Ecological Monograph, 27, 325-349.

Brusca, R. C. \& Findley, L. T. (2005). Listado y distribución de la macrofauna del Golfo de California, México. Tucson, Arizona: Arizona-Sonora Desert Museum.

Calderón, A. A. (2011). Evaluación piloto de los impactos potenciales de las redes de arrastre sobre el ecosistema del Alto Golfo de California durante la temporada de pesca 2010-2011. (Margarita Caso coord.). Convenio $\mathrm{N}^{\circ} \mathrm{NE} / \mathrm{A} 1-038 / 2010$. Baja California. México: Centro de Investigación Científica y de Educación Superior de Ensenada, D.F.

Castro, A. J. L. (1978). Catálogo de peces marinos que penetran a las aguas continentales de México, con aspectos zoogeográticos y Ecológicos. Departamento de Pesca. Instituto Nacional de la Pesca. Serie científica, México, D.F.

Castro, A. J. L., Balart, E. F., \& Arvizu, M. J. (1995). Contribución al conocimiento del origen y distribución de la ictiofauna del Golfo de California, México. Hidrobiológica, 5, 57-78.

Castro, A. J. L. \& Espinosa, P. H. (1996). Listados faunísticos de México. VII. Catálogo sistemático de las rayas y especies afines de México (Chondrichthyes: ElaSPobranchii: Rajiformes: Batoideiomorpha). Instituto de Biología, Universidad Nacional Autónoma de México, México, D.F.

Clarke, K. R., \& Green, R. H. (1988). Statistical design and analysis for a biological effects study. Marine Ecology Progress Series, 46, 213-226.

Clarke, K. R., \& Warwick, R. M. (1998). A taxonomic distinctness index and its statistical properties. Journal of Applied Ecology, 35, 523-531.

Clarke, K. R., \& Warwick, R. M. (1999). The taxonomic distinctness measure of biodiversity: weighting of step lengths between hierarchical levels. Marine Ecology Progress Series, 184, 21-29.

Clarke, K. R., \& Warwick, R. M. (2001). Change in marine communities: An Approach to Statistical Analysis and interpretation. Reino Unido: Plymouth.

Clarke, K. R., \& Gorley, R. N. (2001). PRIMER (Plymouth Routines In Multivariate Ecological Research) v5: User Manual/Tutorial PRIMER-E Plymouth (pp. 91). UK: Plymouth Marine Laboratory.

Clarke, K. R., \& Gorley, R. N. (2006). PRIMER v6: User manual/Tutorial. PRIMER-E Ltd. Plymouth Marine Laboratory, UK. 190 pp.

Compagno, L. J. V. (1984). FAO species catalogue. Vol. 4. Sharks of the world. An annotated and illustrated catalogue of sharks species known to date. Part 1. Hexanchiformes to Lamniformes. FAO Fisheries Synopsis (125) 4, 1, 249 pp.

Cudney, B. R., \& Turk, B. P. J. (1998). Pescando entre mareas del Alto Golfo de California: una guía sobre la pesca artesanal, su gente, y sus propuestas de manejo. Puerto Peñasco, Sonora, México: Centro Intercultural de Estudios de Desiertos y Océanos (CEDO) A.C.

Curran, H. W. (1942). A systematic revision of the gerreid fishes referred to the genus Eucinostomus with a discussion of their distribution and speciation (Ph.D. thesis). University of Michigan, USA.

DOF. (1993). Decreto por el que se declara área natural protegida con el carácter de la Reserva de la Biosfera, la región conocida como Alto Golfo de California y Delta del Río Colorado, ubicada en aguas del Golfo 
de California y los municipios de Mexicali, BC., de Puerto Peñasco y San Luis Río Colorado, Son. México: DOF.

Dumay, O., Tari, P. S., Tomasini, J. A., \& Mouillot, D. (2004). Functional groups of lagoon fish species in Languedoc Roussillon, southern France. Journal of Fish Biology, 64, 970-983.

Escobar, T. F. D. (2012). Variación espacio-temporal de la diversidad de la comunidad de peces asociada a la pesquería de arrastre de camarón del mar caribe de Colombia (Tesis de Doctorado). La Paz, B.C.S., México: CICIMAR-IPN.

Fischer, W., Krupp, F., Schneider, W., Sommer, C., Carpenter, K. E., \& Niem, V. H. (Eds.) (1995). Guía FAO para la identificación de especies para los fines de la pesca. Pacifico Centro-Oriental. Vertebrados. FAO. Roma. 2 (2-3), 648-1652.

Fulton, E. A., Smith, A. D. M., \& Punt, A. E. (2004). Effects of fishing on the structure and functioning of estuarine and nearshore ecosystems. ICES Journal of Marine Science, 57 (3), 590-602.

Froese, R. \& Pauly, D. (2007). FishBase. World Wide Web electronic publication. Retrieved from http://www. fishbase.org

Ginsburg, I. 1958. Flounders of the genus Paralichthys and related genera in American waters. United States Fish and Wildlife Service, Fishery Bulletin, 52, 267-351.

Gristina, M., Bahri, T., Fiorentino, F., \& Garofalo, G. (2006). Comparison of demersal fish assemblages in three areas of the Strait of Sicily under different trawling pressure. Fisheries Research, 81, 60-71.

Hastings, P. A. (2000). Biogeography of the tropical eastern Pacific: distribution and phylogeny of chaenopsid fishes. Zoological Journal of the Linnean Society, $128,319-335$.

Hooper, D. U., Chapin, F. S., Ewel, J. J., Hector, A., Inchausti, P., \& Lavorel, S. (2005). Effects of biodiversity on ecosystem functioning: a consensus of current knowledge. Ecological Monographs, 75, 3-35.

Jordan, D. S., \& Evermann, B. W. (1896-1900). The fishes of North and Middle America. Bulletin Natural Museum, 47, 1-3313.

López, M. J., Hernández, V. S., Herrera, V. E., Rábago, Q. C. H., \& Morales, A. R. (2007). Efectos ecológicos de la pesca de arrastre de camarón en el Golfo de California. Estado del arte del desarrollo Tecnológico de las Artes de Pesca. In CEDRSSA (eds.). $\mathrm{La}$ situación del sector pesquero en México (pp. 14-47). México, D.F.: CEDRSSA y Cámara de diputados LX Legislatura.
López, M. J., Herrera, V. E., Rodríguez, R. J., \& Hernández, V. S. (2010). Composición taxonómica de peces integrantes de la fauna de acompañamiento de la pesca industrial de camarón del Golfo de California, México. Revista de Biología Tropical, 58, 925-942.

López, M. J., Hernández, V. S., Morales, R., Nevárez, M. O., Cervantes, C., \& Padilla, J. (2012). Variación de la relación camarón: fauna de acompañamiento en la pesquería de camarón industrial del Golfo de California. En J. López-Martínez y E. Morales Bojórquez (Eds.), Efectos de la pesca de arrastre en el Golfo de California (25-47 pp.). México: Centro de Investigaciones Biológicas del Noroeste, S.C. y Fundación Produce Sonora, México.

Love, M. S., Yoklavich, M. \& Thorsteinson, L. 2002. The rockfishes of the northeast Pacific. Berkeley, USA: University of California.

Madrid, V. J., Amezcua, F., \& Morales, B. E. (2007). An assesment approach to estimate biomass of fish communities from bycatch data in a tropical shrimp-trawl fishery. Fisheries Research, 83, 81-89.

Mathieson, S., Cattrijsse, A., Costa, M. J., Drake, P., Elliott, M., Gardner, J., \& Marchand, J. (2000). Fish assemblages of European tidal marshes: a comparison based on species, families and functional guilds. Marine Ecology Progress Series, 204, 225-242.

Miller, D. J., \& Lea, R. N. (1972). Guide to the coastal marine fishes of California. California Department of Fish and Game, Fishery Bulletin, 157, 249.

McPhail, J. D. (1958). Key to the croakers (Sciaenidae) of the eastern Pacific (2, 20 p.) Vancouver: Contrib Institute of Fisheries, University of British Columbia.

Nava, R. J. M. (1994). Impactos, a corto y largo plazo, en la diversidad y otras características ecológicas de la comunidad béntico-demersal capturada por la pesquería de camarón en el norte del alto Golfo de California, México (Tesis de Maestría). Instituto Tecnológico y de estudios Superiores de Monterrey, Guaymas, México.

Naeem, S., Lawler, S. P., Thompson, L. J., Lawton, J. H., \&. Woodfin, R. M. (1994). Declining biodiversity can alter the performance of ecosystems. Nature, $368,734-737$.

Nelson, E., Ramírez, E. M., Arenas, F., Carranza, B., Jacquemine, P., Prado, S., \& Solis, N. (1980). Evaluación de los recursos demersales accesibles a redes de arrastre de fondo en el Golfo de California (Mar de Cortés), México, durante 1979. México: Programa de Investigación y Desarrollo Pesquero Integrado. Programa de las Naciones Unidas para el Desarrollo. FAO.

Nelson, J. S. (2006). Fishes of the world. Nueva York: Wiley. 
Nieto, N. J. T. (2010). Estructura y organización de la ictiofauna de fondos blandos del sur de Sinaloa: análisis ecológico y topología de taxa (Tesis de Doctorado). CICIMAR-IPN. La Paz, B.C.S., México.

Norman, J. R. (1934). A systematic monograph of the flat fishes (Heterostomata). Psettedidae, Bothidae, Pleuronectidae. London British Museum (Natural History), 1, 459.

British.

Palacios-Salgado, D. (2011). Patrones Latitudinales de composición y Diversidad funcional de peces asociados a la pesca de camarón del Pacífico Mexicano (Tesis de Doctorado). CICIMAR-IPN. La Paz, B.C.S., México.

Pérez, M. J., \& Findley, L. T. (1985). Evaluación de la ictiofauna acompañante del camarón comercial capturado en las costas de Sonora y norte de Sinaloa. En A. Yáñez-Arancibia (Ed.), Recursos pesqueros potenciales de México: La pesca acompañante del camarón (Cap. 5, pp. 201-254). UNAM, México DF.: Programa Universitario De Alimentos, Instituto Ciencias Del Mar y Limnología, Instituto Nacional Pesca.

Rábago, Q. C. H., López, M. J., Herrera, V. E., Nevárez, M. M. O., \& Rodríguez, R. (2010). Population dynamics and spatial distribution of flatfish species in shrimp trawl bycatch in the Gulf of California. Hidrobiológica, 18(2), 177-188.

Rábago, Q. C. H., López, M. J., Valdez, H. J. E., \& Nevárez, M. M. O. (2011). Distribución latitudinal y batimétrica de las especies más abundantes y frecuentes en la fauna acompañante del camarón del Golfo de California, México. Revista de Biología Tropical, 59(1), 255-267.

Ramos, M., Mouillot, M. J. D., Flores, D., Sosa, A., Do Chi, T. \& Ayala, L. (2005). Changes in four complementary facets of fish diversity in a tropical coast lagoon after 18 years: a functional interpretation. Marine Ecology Progress Series, 304, 1-13.

Robertson, D. R., \& Allen, G. R. (2002). Shore fishes of the Tropical Eastern Pacific: an information system. Balboa, Panamá: Smithsonian Tropical Research Institute.

Robertson, D. R., \& Allen, G. R. 2015. Peces Costeros del Pacifico Oriental Tropical: sistema de Información en línea. Versión 2.0 Instituto Smithsonian de Investigaciones Tropicales, Balboa, República de Panamá.
Downloaded from http://biogeodb.stri.si.edu/sftep/ es/pages).

Rosenblatt, R. H. \& Johnson, G. D. 1974. Two new species of sea basses of the genus Diplectrum, with a key to the Pacific species. California Fish and Game, 60, 178-191.

Rosenfeld, J. S. (2002). Functional redundancy in ecology and conservation. Oikos, 98(1), 156-162.

Somerfield, P. J., Clarke, K. R., Warwic, R. M. K., \& Dulvy, N. K. (2008). Average functional distinctness as a measure of the composition of assemblages. ICES Journal of Marine Science, 65, 1462-1468.

Tilman, D. (2001). Functional diversity. In S. A. Levin (Ed.), Encyclopedia of biodiversity (pp. 109-120). Academic Press.

Valenzuela, T. M., Herrera, E. V., \&. Gracia, S. N. (1988). Evaluación de la fauna que acompaña al camarón en arrastres comerciales de las costas de Sonora y Sinaloa, México. (Tesis de Licenciatura). Universidad Autónoma de Sinaloa, Mazatlán, Sinaloa.

Walker, H. J., \& Rosenblatt, R. H. (1988). Pacific toadfish hes of the genus Porichthys (Batrachoididae) with descriptions of three new species. Copeia, 4, 887-904.

Walker, B., Holling, C. S., Carpenter, S. R. \& Kinzig, A. (2004). Resilience, adaptability and transformability in social-ecological systems. Ecology and Society 9, 5. Tomado de http://www.ecologyandsociety.org/ vol9/iss2/art5/

Warwick, R. M., \& Clarke, K. R. (1994). Relearning the $\mathrm{ABC}$ : taxonomic changes and abundance/biomass relationships in disturbed benthic communities. Marine Biology, 118, 739-744.

Warwick, R. M., \& Clarke, K. R. (1995). New 'biodiversity' measures reveal a decrease in taxonomic distinctness with increasing stress. Marine Ecology Progress Series. 129, 301-305.

Warwick, R. M., \& K. R., Clarke. (1998). Taxonomic distinctness and environmental assessment. Journal of Applied Ecology, 35, 532-543.

Zahuranec, J. B. (1967). The gerreid fishes of the genus Eucinostomus in the Eastern Pacific (Tesis de Maestría). Universidad de California, San Diego, California. 